
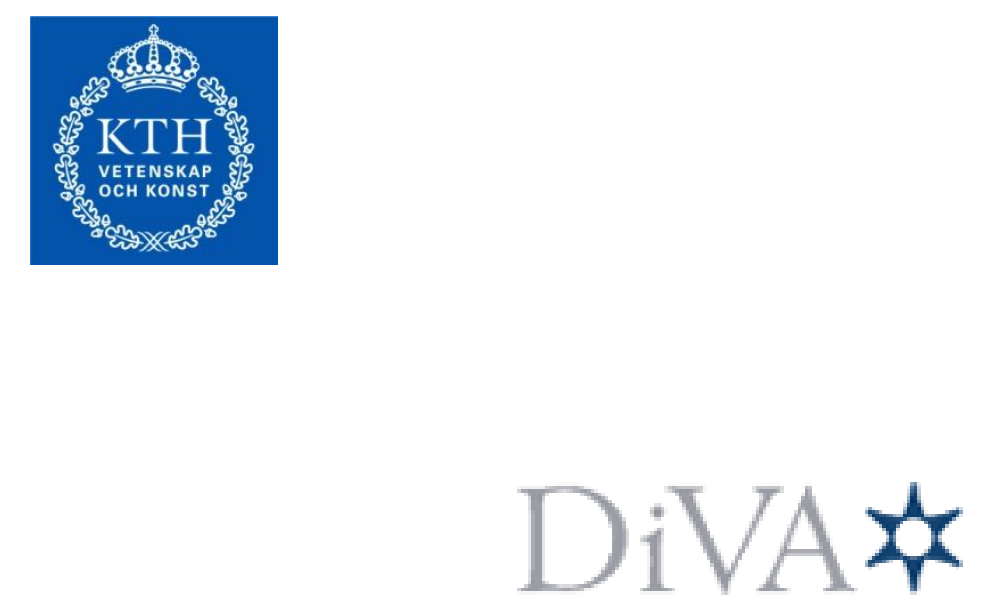

http://kth.diva-portal.org

This is the author's version of a work that was accepted for publication in Automatica. Changes resulting from the publishing process, such as peer review, editing, corrections, structural formatting, and other quality control mechanisms may not be reflected in this document. Changes may have been made to this work since it was submitted for publication. A definitive version was subsequently published in Automatica, VOL 48, ISSUE 10, (October 2012) DOI 10.1016/j.automatica.2012.06.067

Access to the published version may require subscription. Publish with permission from: Elsevier 


\title{
Finite model order accuracy in Hammerstein model estimation
}

\author{
Håkan Hjalmarsson ${ }^{\mathrm{a}}$ Jonas Mårtensson ${ }^{\mathrm{a}}$ \\ a ACCESS Linnaeus Center, Electrical Engineering, \\ KTH Royal Institute of Technology, SE-100 44 Stockholm, Sweden
}

\begin{abstract}
Hammerstein models is one of the most commonly used model classes used for identifying nonlinear systems. A static input nonlinearity followed by a linear dynamical part is an adequate way to model many real-life systems. This paper investigates the asymptotic (in terms of sample size) variance of Hammerstein model estimates. The work extends earlier results by Ninness and Gibson (2002) in the following ways. Not only frequency function estimation but estimation of general quantities is considered. The expressions are not restricted to be valid asymptotically in the model order. In addition, the results cover model structures having noise models and allow for data generated under feedback. The increase in variance due to the estimation of the input nonlinearity is characterized. In particular, under open loop operation, white additive noise and the assumption of a separable process, it is shown that the variance increase is exactly a term that was observed in Ninness and Gibson (2002) to result in good agreement with simulations. This term vanishes in the formal asymptotic in model order analysis in Ninness and Gibson (2002).
\end{abstract}

\section{Introduction}

Hammerstein models have found widespread use in a range of disciplines, e.g. process control (Eskinat et al.; 2004) and biological systems (Hunter and Korenberg; 1986). A wide range of identification methods have also been developed for this type of model structure, see e.g. Bai (1998); Greblicki and Pawlak (1986); Goethals et al. (2005); Zhu (2000); Crama and Schoukens (2001); Ding and Chen (2005); Cai and Bai (2011); Vanbeylen et al. (2008); Wang et al. (2009); Liu and Bai (2007).

However, the accuracy of an estimated Hammerstein model has received very limited attention. One exception is Ninness and Gibson (2002) where an asymptotic, in the model order, expression for the variance of the estimate of the linear part of the model is derived. Through this expression it is revealed that the nonlinear part of the model contribute to the variance of the linear part of the model.

In this contribution we derive conditions for when the variance expression derived in Ninness and Gibson (2002) is valid for finite model orders. We also extend the result to hold for colored noise. Preliminary results on this have been presented in Hjalmarsson and

\footnotetext{
* This work was supported in part by the Swedish Research Council under contracts 621-2007-6271 and 621-2009-4017.
}

Mårtensson (2007).

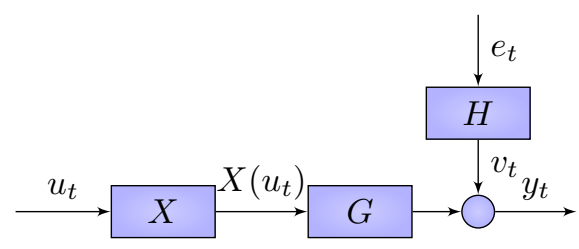

Fig. 1. Block diagram of a Hammerstein model. The block $X$ is a static nonlinearity whereas $G$ and $H$ are linear dynamic blocks.

\section{A Prediction error framework and assump-} tions

\subsection{The Hammerstein model}

A Hammerstein model structure, depicted in Figure 1, is given by

$$
\begin{aligned}
y_{t} & =G(\mathrm{q}, \beta) x_{t}(\alpha)+H(\mathrm{q}, \beta) e_{t}, \\
x_{t}(\alpha) & =X\left(u_{t}, \alpha\right)
\end{aligned}
$$

where $X$ is a memoryless non-linearity, continuously differentiable in the parameter vector $\alpha \in \mathbb{R}^{n_{\alpha}}$.

The linear time-invariant (LTI) single-input singleoutput (SISO) dynamical model $G$ and the LTI SISO 
noise model $H$ are rational functions in $\mathrm{q}$ and are parameterized by the vector $\beta \in \mathbb{R}^{n_{\beta}}$. We assume that $G$ contains at least one pure time delay. The noise model is monic and inversely stable. The parameters are collected in

$$
\theta:=\left[\begin{array}{ll}
\beta^{T} & \alpha^{T}
\end{array}\right]^{T} \in \mathbb{R}^{n_{\beta}+n_{\alpha}}
$$

We will assume that the model set contains the true system, i.e. there exists a model parameter $\theta_{o}=\left[\begin{array}{cc}\beta_{o}^{T} & \alpha_{o}^{T}\end{array}\right]^{T}$ such that (1) is a correct description of the data generating mechanism when $\theta=\theta_{o}$. Furthermore, we assume that the model is identifiable at $\theta_{o}$. We assume that $G_{O}$ is stable. We also assume that the true noise sequence $\left\{e_{t}^{o}\right\}$ is an i.i.d., zero mean white noise process with variance $\lambda_{o}$ and that the input $\left\{u_{t}\right\}$ is a stationary stochastic process with finite variance.

Remark: It is common to have Hammerstein parameterizations where both $G$ and $X$ can be scaled by constant factors. In that case identifiability is lost since $G$ can be multiplied by a constant and $X$ be divided by the same constant without changing the product of $G$ and $X$. This is, however, easily fixed by changing the parametrization, e.g, by fixing some parameter in either $G$ or $X$. That parameter would have to be known to be non-zero, but that is usually not such a severe limitation. Fixing the first coefficient in the numerator of a polynomial transfer function model, for example, would mean that the number of pure time delays in the system is known. Another option would be to normalize the norm of the parameter vector, for example requiring that the norm of the sub-vector that contains the numerator coefficients is equal to one.

The following notation will be used throughout the paper.

Notation 2.1 In some cases we will use the notation $T(\mathrm{q}, \beta):=[G(\mathrm{q}, \beta), H(\mathrm{q}, \beta)]^{T}$ and $z_{t}(\alpha):=\left[x_{t}(\alpha), e_{t}\right]^{T}$. Furthermore, $G_{o}(\mathrm{q}):=G\left(\mathrm{q}, \beta_{o}\right), H_{o}(\mathrm{q}):=H\left(\mathrm{q}, \beta_{o}\right)$, $T_{o}(\mathrm{q}):=T\left(\mathrm{q}, \beta_{o}\right), x_{t}^{o}:=x_{t}\left(\alpha_{o}\right)$ and $z_{t}^{o}:=z_{t}\left(\alpha_{o}\right)$. For derivatives we use the notation $T^{\prime}(\mathrm{q})=\left[G^{\prime}(\mathrm{q}), H^{\prime}(\mathrm{q})\right]$ where $G^{\prime}(\mathrm{q}):=\left.\frac{\partial G(\mathrm{q}, \beta)}{\partial \beta}\right|_{\beta=\beta_{o}}, H^{\prime}(\mathrm{q}):=\left.\frac{\partial H(\mathrm{q}, \beta)}{\partial \beta}\right|_{\beta=\beta_{o}}$, and $x_{t}^{\prime}:=\partial x_{t}(\alpha) /\left.\partial \alpha\right|_{\alpha=\alpha_{o}}$, where the gradients are column vectors. Generally, for a differentiable function $f$ : $\mathbb{C}^{n} \rightarrow \mathbb{C}^{m}$ we will use $f^{\prime}(\bar{x})$ to denote the $n \times m$ matrix with $\left.\frac{\partial f_{j}(x)}{\partial x_{i}}\right|_{x=\bar{x}}$ as ij:th entry. Finally, $\bar{x}$ and $x^{*}$ denote the complex conjugate and the complex conjugate transpose of $x$, respectively.

\subsection{Prediction error identification}

We will assume that the model parameters $\theta$ are estimated by way of prediction error identification. For sim- plicity we consider a quadratic cost function

$$
V_{N}(\theta)=\frac{1}{2 N} \sum_{t=1}^{N} \varepsilon_{t}^{2}(\theta)
$$

where the prediction error is given by

$$
\varepsilon_{t}(\theta)=H^{-1}(\mathrm{q}, \beta)\left(y_{t}-G(\mathrm{q}, \beta) x_{t}(\alpha)\right)
$$

The parameter estimate $\hat{\theta}_{N}=\left[\begin{array}{ll}\hat{\beta}_{N}^{T} & \hat{\alpha}_{N}^{T}\end{array}\right]^{T}$ is defined by

$$
\hat{\theta}_{N}:=\arg \min V_{N}(\theta)
$$

\subsection{Asymptotic estimation error covariance}

It will be assumed that the estimate is consistent, i.e. it holds that

$$
\hat{\theta}_{N} \rightarrow \theta_{o} \text { w.p.1 }
$$

and that the estimate is asymptotically normal distributed

$$
\sqrt{N}\left(\hat{\theta}_{N}-\theta_{o}\right) \in A s N(0, P)
$$

where

$$
P=\left[\begin{array}{cc}
P_{\beta} & P_{\beta \alpha} \\
P_{\beta \alpha}^{T} & P_{\alpha}
\end{array}\right], P_{\beta} \in \mathbb{R}^{n_{\beta} \times n_{\beta}}, P_{\alpha} \in \mathbb{R}^{n_{\alpha} \times n_{\alpha}}
$$

In $(5), \operatorname{AsN}(m, P)$ denotes that the left-hand side converges in distribution to the normal distribution with mean $m$ and covariance matrix $P$, as $N$ goes to infinity.

The exact conditions for when (4) and (5) hold are outside the scope of the paper. Bauer and Ninness (2002) provides an analysis of both consistency and asymptotic normality for least-squares estimation of HammersteinWiener models. One of the conditions is identifiability of the model, i.e. that $\varepsilon_{t}\left(\theta_{1}\right)=\varepsilon_{t}\left(\theta_{2}\right)$ for all $t$ if and only if $\theta_{1}=\theta_{2}$. The issue here is to establish appropriate conditions for persistence of excitation with respect to the particular model. In Zhao (2010), the consistency of an extended least squares estimate is shown for the case when the linear part is an ARMAX model and nonlinearity is a linear combination of basis functions. The input is an i.i.d. random variable with a continuous probability density function with finite support. Similar analysis can be performed for the the settings considered in this paper, but that will not be pursued here. It is, however, quite straightforward to show identifiability for an odd polynomial input nonlinearity with white output noise 
$(H=1)$ and a white zero-mean input process with symmetric continuous probability density function with finite support. Identifiability can also be shown for the same case but with zero-mean Gaussian input.

In Ljung and Caines (1979) it is shown that the asymptotic covariance matrix can be written as

$$
P=\lambda_{o}\left[\mathrm{E}\left[\Psi_{t} \Psi_{t}^{\mathrm{T}}\right]\right]^{-1}
$$

where $\Psi_{t}$ is the negative gradient of the prediction error (2) evaluated at $\theta_{o}$, i.e.

$$
\Psi_{t}=\left[\begin{array}{cc}
\frac{1}{H_{o}(\mathrm{q})} T^{\prime}(\mathrm{q}) & 0_{n_{\beta} \times n_{\alpha}} \\
0_{n_{\alpha} \times 2} & \frac{G_{o}(\mathrm{q})}{H_{o}(\mathrm{q})} I_{n_{\alpha} \times n_{\alpha}}
\end{array}\right]\left[\begin{array}{c}
z_{t}^{o} \\
x_{t}^{\prime}
\end{array}\right]
$$

With $J(\theta): \mathbb{R}^{n} \rightarrow \mathbb{C}^{n_{J}}$, we define the asymptotic covariance of $J\left(\hat{\theta}_{N}\right)$ as

$$
\begin{aligned}
& \operatorname{AsCov} J\left(\hat{\theta}_{N}\right)= \\
& \lim _{N \rightarrow \infty} N \cdot \mathrm{E}\left[\left(J\left(\hat{\theta}_{N}\right)-J\left(\theta_{o}\right)\right)\left(J\left(\hat{\theta}_{N}\right)-J\left(\theta_{o}\right)\right)^{*}\right]
\end{aligned}
$$

if the limit above is well defined. When $J$ is scalar valued, we will define the asymptotic variance $\operatorname{AsVar} J\left(\hat{\theta}_{N}\right)$ of $J\left(\hat{\theta}_{N}\right)$ as in $(8)$.

When $J$ is differentiable, Gauss' approximation formula (Lehmann; 1983) gives that

$$
\operatorname{AsCov} J\left(\hat{\theta}_{N}\right)=\Lambda^{T} P \bar{\Lambda}
$$

where

$$
\Lambda:=J^{\prime}\left(\theta_{o}\right)
$$

The starting point for the analysis in this paper will be (9).

Remark: The expressions (6) and (9) correspond to the asymptotic Cramér-Rao lower bound, which means that the asymptotic covariance cannot be less than (9) for any consistent estimator of $J\left(\hat{\theta}_{N}\right)$. It is well known that the least-squares estimator (3) achieves that bound asymptotically.

For the upcoming analysis we need to introduce some more notation.

Notation 2.2 For two functions $f, g: \mathbb{C} \rightarrow \mathbb{C}^{n \times m}$ we use $\langle f, g\rangle$ to denote the integral

$$
\langle f, g\rangle=\frac{1}{2 \pi} \int_{-\pi}^{\pi} f\left(\mathrm{e}^{\mathrm{j} \omega}\right) g^{*}\left(\mathrm{e}^{\mathrm{j} \omega}\right) \mathrm{d} \omega
$$

A function $\Psi: \mathbb{C} \rightarrow \mathbb{C}^{n \times m}$ is said to belong to $\mathcal{L}_{2}^{n \times m}$ when $\langle\Psi, \Psi\rangle$ exists. We write $\Psi \in \mathcal{L}_{2}^{n \times m}$ for this. For a $\Psi \in \mathcal{L}_{2}^{n \times m}, \mathcal{S}_{\Psi}$ is the subspace to $\mathcal{L}_{2}^{m}$ spanned by the rows of $\Psi$. Furthermore, for a $\gamma \in \mathcal{L}_{2}^{n_{J} \times m}, \mathbf{P}_{\mathcal{S}_{\Psi}}\{\gamma\}$ denotes the orthogonal projection of $\gamma$ onto the subspace $\mathcal{S}_{\Psi}$. With $\mathcal{X}, \mathcal{Y}$ being closed subspaces of a Hilbert space, we use $\mathcal{X}+\mathcal{Y}$ to denote the subspace $\{x+y: x \in \mathcal{X}, y \in \mathcal{Y}\}$, $\mathcal{Z}=\mathcal{X} \oplus \mathcal{Y}$ to denote that $\mathcal{Z}$ is the direct sum of $\mathcal{X}$ and $\mathcal{Y}$, i.e. that $\mathcal{Y}$ is the orthogonal complement of $\mathcal{X}$ in $\mathcal{Z}$. Furthermore, we define $\mathcal{Y} \ominus \mathcal{X}$ to be the orthogonal complement of $\mathcal{X}$ in $\mathcal{X}+\mathcal{Y}$, i.e. $\mathcal{X}+\mathcal{Y}=\mathcal{X} \oplus(\mathcal{Y} \ominus \mathcal{X})$.

\subsection{A geometric covariance expression}

When $P$ in (5) has the structure $P=\langle\Psi, \Psi\rangle^{-1}$ for some $\Psi: \mathbb{C} \rightarrow \mathbb{C}^{n \times m}$ (for some positive integer $m$ ), so that (9) can be written

$$
\operatorname{AsCov} J\left(\hat{\theta}_{N}\right)=\Lambda^{T}\langle\Psi, \Psi\rangle^{-1} \bar{\Lambda}
$$

the following result holds.

Lemma 2.1 Suppose that $J: \mathbb{R}^{n} \rightarrow \mathbb{C}^{n_{J}}$ is differentiable and suppose that the asymptotic covariance matrix AsCov $J\left(\hat{\theta}_{N}\right)$ is given by (11) for some $\Psi \in \mathcal{L}_{2}^{n \times m}$ such that $\langle\Psi, \Psi\rangle>0$. Suppose that $\gamma \in \mathcal{L}_{2}^{n_{J} \times m}$ is such that

$$
\Lambda=\langle\Psi, \gamma\rangle
$$

Then

$$
\operatorname{AsCov} J\left(\hat{\theta}_{N}\right)=\left\langle\mathbf{P}_{\mathcal{S}_{\Psi}}\{\gamma\}, \mathbf{P}_{\mathcal{S}_{\Psi}}\{\gamma\}\right\rangle^{\mathrm{T}}
$$

In particular, when $J$ is scalar,

$$
\operatorname{As} \operatorname{Var} J\left(\hat{\theta}_{N}\right)=\left\|\mathbf{P}_{\mathcal{S}_{\Psi}}\{\gamma\}\right\|^{2}
$$

Proof: This is Theorem II.5 in Hjalmarsson and Mårtensson (2011).

The relations (12)-(14) provide a geometric interpretation of the asymptotic covariance matrix. Expression (14) shows that the asymptotic variance of $J\left(\hat{\theta}_{N}\right)$ is given by the squared norm of the projection of $\gamma$ onto the subspace spanned by the rows of $\Psi$.

In certain cases it is easy to compute the projection $\mathbf{P}_{\mathcal{S}_{\Psi}}\{\gamma\}$ explicitly. Some of our contributions rely on the following lemma.

Lemma 2.2 Let $J, \Psi$ and $\mathcal{S}_{\Psi}$ be as in Lemma 2.1 and suppose that $\Lambda=\Psi\left(z_{o}\right) L$ for some $z_{o} \in \mathbb{C}$ and $L \in$ 
$\mathbb{C}^{m \times n_{J}}$. Let $\left\{\mathcal{B}_{k}\right\}_{k=1}^{r}, r \leq n$, be an orthonormal basis for $\mathcal{S}_{\Psi}$. Then (13) can be expressed as

$$
\operatorname{AsCov} J\left(\hat{\theta}_{N}\right)=L^{\mathrm{T}} \sum_{k=1}^{r} \mathcal{B}_{k}^{\mathrm{T}}\left(z_{o}\right) \overline{\mathcal{B}_{k}\left(z_{o}\right)} \bar{L}
$$

Proof: This is Lemma II.9 in Hjalmarsson and Mårtensson (2011).

These variance expressions were used for analyzing the accuracy of error-in-variables identification in Hjalmarsson et al. (2011) and here they are used for analyzing the accuracy of Hammerstein model identification.

\section{Covariance of Hammerstein models}

Let $\Phi_{z}$ denote the spectrum of a signal $z$ and $\Phi_{z w}$ be the cross-spectrum between two signals $z$ and $w$. Parseval's formula gives that (6) can be expressed as

$$
\operatorname{AsCov} \hat{\theta}_{N}=\langle\Psi, \Psi\rangle^{-1}
$$

where

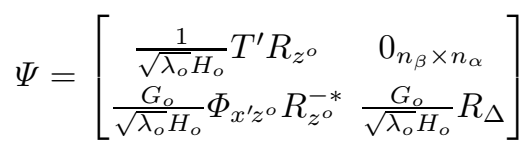

Above, $R_{z^{\circ}}$ and $R_{\Delta}$ are stable spectral factors of $\Phi_{z^{\circ}}$ and $\Phi_{\Delta}$, respectively, where

$$
\Phi_{\Delta}:=\Phi_{x^{\prime}}-\Phi_{x^{\prime} z^{o}} \Phi_{z^{o}}^{-1} \Phi_{z^{o} x^{\prime}}
$$

The derivation of (15)-(17) is straightforward and the details can be found in Appendix A.

The geometric interpretation in Lemma 2.1 now leads to the following result for the covariance of Hammerstein models.

Theorem 3.1 Let $\Psi$ be given by (16) and let $J^{\prime}\left(\theta_{o}\right)=$ $\langle\Psi, \gamma\rangle$ for some $\gamma \in \mathcal{L}_{2}^{n_{J} \times m}$.

Then

$$
\operatorname{AsCov} J\left(\hat{\theta}_{N}\right)=C_{1}+C_{2}
$$

where

$$
\begin{aligned}
& C_{1}=\left\langle\mathbf{P}_{\mathcal{S}_{\Psi_{1}}}\{\gamma\}, \mathbf{P}_{\mathcal{S}_{\Psi_{1}}}\{\gamma\}\right\rangle^{T} \\
& C_{2}=\left\langle\mathbf{P}_{\mathcal{S}_{\Psi_{2}} \ominus \mathcal{S}_{\Psi_{1}}}\{\gamma\}, \mathbf{P}_{\mathcal{S}_{\Psi_{2}} \ominus \mathcal{S}_{\Psi_{1}}}\{\gamma\}\right\rangle^{T}
\end{aligned}
$$

where $\Psi_{1}$ and $\Psi_{2}$ denote the first and second block row of $\Psi$, respectively.
Proof: The result is a direct consequence of Lemma 2.1 and the orthogonal decomposition $\mathcal{S}_{\Psi}=\mathcal{S}_{\Psi_{1}}+\mathcal{S}_{\Psi_{2}}=$ $\mathcal{S}_{\Psi_{1}} \oplus\left(\mathcal{S}_{\Psi_{2}} \ominus \mathcal{S}_{\Psi_{1}}\right)$.

The first term above, $C_{1}$, corresponds to the covariance of $J\left(\hat{\theta}_{N}\right)$ in the case when the non-linear part $X\left(u_{t}, \alpha\right)$ is known and the linear part is estimated from $x_{t}^{o}$ and $y_{t}$. The second term, $C_{2}$, represents the increase of the covariance when also the non-linearity $X\left(u_{t}, \alpha\right)$ has to be estimated.

\subsection{Separable processes}

Further assumptions on the signals will be imposed to derive more explicit expressions for the term $C_{2}$ in (19) above. For this we need to introduce the notion of separable processes.

Definition 3.2 (Separable processes) A stationary stochastic process $\left\{v_{t}\right\}$ with $\mathrm{E}\left[v_{t}\right]=0$ (zero expectation) is separable if

$$
\mathrm{E}\left[v_{t-\tau} \mid v_{t}\right]=a_{\tau} v_{t}
$$

for some sequence $\left\{a_{\tau}\right\}$. The separability notion is adopted from Nuttall (1958), where also this condition can be found.

The sequence $\left\{a_{\tau}\right\}$ in Definition 3.2 can be expressed as

$$
a_{\tau}=\frac{r_{v}(\tau)}{r_{v}(0)}
$$

where $r_{v}$ is the autocorrelation of $\left\{v_{t}\right\}$, i.e. $r_{v}(\tau)=$ $\mathrm{E}\left[v_{t-\tau} v_{t}\right]$, see Nuttall (1958); Enqvist (2005) for details.

White noise, Gaussian processes and random multi-sines are examples of separable processes (Enqvist; 2005).

We will use the following result.

Lemma 3.3 Let $\left\{v_{t}\right\}$ be a zero mean stationary separable process with spectrum $\Phi$ and let $f$ be a static nonlinearity $f$ such that $f\left(v_{t}\right)$ is stationary with zero mean and for which $\mathrm{E}\left[f\left(v_{t}\right) v_{t-\tau}\right]$ exists for all $\tau$. It then holds that the cross-spectrum between $f(u)$ and $u$ is given by $\delta \Phi$ for some constant $\delta>0$.

Proof: The proof can be found in Nuttall (1958); Enqvist (2005).

Under the assumption that $\left\{x_{t}^{o}\right\}$ is a separable process, we have the following result.

Theorem 3.4 Let $\Psi$ and $\gamma$ be as in Theorem 3.1. Impose the following assumptions:

i. $\left\{x_{t}^{o}\right\}$ is a separable process 
ii. $x_{t}^{\prime}=f\left(x_{t}^{o}\right)$ for some static nonlinearity $f(\cdot)$ that fulfills the conditions of Lemma 3.3.

iii. $\left\{u_{t}\right\}$ and $\left\{e_{t}^{o}\right\}$ are uncorrelated, i.e. $\Phi_{u e}=0$

iv. $\left[G_{o} 0\right] \in \mathcal{S}_{\tilde{\Psi}_{1}}, \quad \tilde{\Psi}_{1}=\left[G^{\prime} \frac{1}{R_{x^{o}}} H^{\prime}\right]$

Then $C_{2}$ in (18) is given by

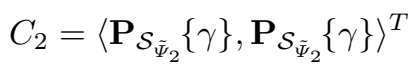

where

$$
\tilde{\Psi}_{2}=\left[0 \frac{G_{o}}{H_{o}} R_{\Delta}\right]
$$

Proof: See Appendix B.

Condition (ii) holds, for example, when $X\left(u, \alpha_{o}\right)$ has an inverse $g$ with respect to $u$, that is, $g\left(X\left(u, \alpha_{o}\right)\right)=u$. Then we can write

$$
x_{t}^{\prime}=\frac{\partial X\left(u_{t}, \alpha_{o}\right)}{\partial \alpha}=\frac{\partial X\left(g\left(x_{t}\left(\alpha_{o}\right)\right), \alpha_{o}\right)}{\partial \alpha}
$$

that is, we can view $x_{t}^{\prime}$ as a function of $x_{t}\left(\alpha_{o}\right)$. For condition (iii) to hold, there can be no feedback from $y$ to $u$ so the system has to operate in open loop. When $H(\mathrm{q}, \beta)=1$ (not estimated), then the entire column corresponding to $e_{t}$ vanishes from the expressions and condition (iii) is no longer needed. Also, under this condition $\Phi_{\Delta}(17)$ reduces to

$$
\Phi_{\Delta}:=\Phi_{x^{\prime}}-\frac{\Phi_{x^{o} x^{\prime}}^{*} \Phi_{x^{o} x^{\prime}}}{\Phi_{x^{o}}}
$$

Condition (iv) in Theorem 3.4 typically holds for many model structures, including ARMAX, Box-Jenkins and output-error models. If the parameters of $B(\mathrm{q}, \beta)$ (the numerator polynomial of $G(\mathrm{q}, \beta)=B(\mathrm{q}, \beta) / A(\mathrm{q}, \beta))$ does not share parameters with $H(\mathrm{q}, \beta)$, then the rows of $\tilde{\Psi}_{1}$ that correspond to the parameters in $B$ are given by $\left[\frac{1}{A_{o}} B^{\prime} 0\right]$ and condition (iv) will be fulfilled if $B_{o} \in \mathcal{S}_{B^{\prime}}$. When $G$ and $H$ are independently parameterized, or when $H$ is not estimated, the condition (iv) reduces to that $G_{o} \in \mathcal{S}_{G^{\prime}}$.

\section{Covariance of the linear part}

The asymptotic covariance of the frequency function estimate of the linear part of the model and the noise model, $T\left(\mathrm{e}^{\mathrm{j} \omega}, \hat{\beta}_{N}\right)$, is given by (9) with

$$
\Lambda=\left[\begin{array}{c}
T^{\prime}\left(\mathrm{e}^{\mathrm{j} \omega_{o}}\right) \\
0_{n_{\alpha} \times 2}
\end{array}\right]
$$

Before we proceed and analyze the asymptotic covariance of $T\left(\mathrm{e}^{\mathrm{j} \omega}, \hat{\beta}_{N}\right)$ with the geometric tools introduced above, we review an existing result by Ninness and Gibson (2002).

For this result we introduce the following notation. Let $\frac{\mathrm{d}}{\mathrm{d} \alpha} \log \Phi_{x}\left(\omega_{o}\right)$ be short-hand for the partial derivative of the logarithm of the spectrum of $x_{t}(\alpha)$ with respect to $\alpha$, evaluated at $\alpha_{o}$.

Result 4.1 (Ninness and Gibson (2002)) For the case of white output noise, i.e. when $H(\mathrm{q}, \beta)=1$, and when the system dynamics is modeled as a function expansion

$$
G(\mathrm{q}, \beta)=\sum_{k=1}^{n_{\beta}} \beta_{k} \mathcal{B}_{k}^{\mathcal{M}}(\mathrm{q})
$$

where $\left\{\mathcal{B}_{k}^{\mathcal{M}}\right\}$ is a set of orthonormal basis functions, it is shown in Ninness and Gibson (2002) that AsVar $G\left(\mathrm{e}^{\mathrm{j} \omega_{o}}, \hat{\beta}_{N}\right)$ behaves in the following way as the model order increases:

$$
\begin{aligned}
& \lim _{n_{\beta} \rightarrow \infty} \frac{1}{K_{n_{\beta}}\left(\omega_{o}\right)} \operatorname{As} \operatorname{Var} G\left(\mathrm{e}^{\mathrm{j} \omega_{o}}, \hat{\beta}_{N}\right)=\frac{\lambda_{o}}{\Phi_{x^{o}}\left(\omega_{o}, \alpha_{o}\right)}+ \\
& \frac{1}{4}\left(\frac{\mathrm{d}}{\mathrm{d} \alpha} \log \Phi_{x}\left(\mathrm{e}^{\mathrm{j} \omega_{o}}\right)\right)^{\mathrm{T}} P_{\alpha}\left(\frac{\mathrm{d}}{\mathrm{d} \alpha} \log \Phi_{x}\left(\mathrm{e}^{\mathrm{j} \omega_{o}}\right)\right) \\
& \times \lim _{n_{\beta} \rightarrow \infty} \frac{\left|G\left(\mathrm{e}^{\mathrm{j} \omega_{o}}, \beta_{o}\right)\right|^{2}}{K_{n_{\beta}}\left(\omega_{o}\right)}
\end{aligned}
$$

where

$$
K_{n_{\beta}}(\omega):=\sum_{k=1}^{n_{\beta}}\left|\mathcal{B}_{k}^{\mathcal{M}}\left(\omega_{o}\right)\right|^{2}
$$

and

$$
P_{\alpha}=\lambda_{o}\left\langle G_{o} R_{\Delta}, G_{o} R_{\Delta}\right\rangle^{-1}
$$

One interesting observation in Ninness and Gibson (2002) is that the non-linear part of the model increases the asymptotic variance of $G\left(\mathrm{e}^{\mathrm{j} \omega_{o}}, \hat{\beta}_{N}\right)$, at least as the model order grows. This corresponds to the second term of the right-hand side of (27).

Actually, it can be shown, see Ninness et al. (1999), that $K_{n_{\beta}}\left(\omega_{o}\right) \rightarrow \infty$ as $n_{\beta} \rightarrow \infty$ and hence formally the result (27) can be re-stated as

$$
\lim _{n_{\beta} \rightarrow \infty} \frac{1}{K_{n_{\beta}}\left(\omega_{o}\right)} \operatorname{As} \operatorname{Var} G\left(\mathrm{e}^{\mathrm{j} \omega_{o}}, \hat{\beta}_{N}\right)=\frac{\lambda_{o}}{\Phi_{x^{o}}\left(\omega_{o}, \alpha_{o}\right)}
$$

i.e., the second term of the right-hand side of (27) is zero. However, in Ninness and Gibson (2002) it is observed 
that this term improves the accuracy of the variance expression. In particular, in Ninness and Gibson (2002, Section 5) it is shown that including this term results in very good agreement with Monte Carlo simulations for both a saturation and a dead-zone example where the input is white noise in both cases.

We will now use the geometric tools to study this result for finite model orders.

\subsection{Expressions for finite model orders}

We can write $\Lambda$ (as defined in (25)) as $\Psi\left(\mathrm{e}^{\mathrm{j} \omega_{o}}\right) L\left(\mathrm{e}^{\mathrm{j} \omega_{o}}\right)$, where

$$
\begin{aligned}
& L\left(\mathrm{e}^{\mathrm{j} \omega_{o}}\right) \\
& =\left[\begin{array}{c}
\sqrt{\lambda_{o}} H_{o}\left(\mathrm{e}^{\mathrm{j} \omega_{o}}\right) R_{z^{o}}^{-1}\left(\mathrm{e}^{\mathrm{j} \omega_{o}}\right) \\
-\sqrt{\lambda_{o}} H_{o}\left(\mathrm{e}^{\mathrm{j} \omega_{o}}\right) R_{\Delta}^{-1}\left(\mathrm{e}^{\mathrm{j} \omega_{o}}\right) \Phi_{x^{\prime} z^{o}}\left(\mathrm{e}^{\mathrm{j} \omega_{o}}\right) \Phi_{z^{o}}^{-1}\left(\mathrm{e}^{\mathrm{j} \omega_{o}}\right)
\end{array}\right]
\end{aligned}
$$

Lemma 2.2 now gives the following result.

Theorem 4.2 Let $\Psi, \Psi_{1}, \Psi_{2}$ and $\gamma$ be as in Theorem 3.1. Let $\left\{\mathcal{B}_{k}^{\beta}\right\}_{k=1}^{n_{\beta}}$ be an orthonormal basis for the rowspace of $H_{o}^{-1} T^{\prime} R_{z}$ and let $\left\{\mathcal{B}_{k}^{\alpha}\right\}_{k=1}^{n_{\alpha}}$ be an orthonormal basis for $\mathcal{S}_{\Psi_{2}} \ominus \mathcal{S}_{\Psi_{1}}$. Then the asymptotic covariance matrix of $T\left(\mathrm{e}^{\mathrm{j} \omega_{o}}, \hat{\beta}_{N}\right)$ as defined by (9), with $\Lambda$ given by (25), can be expressed as

$$
\operatorname{AsCov} T\left(\mathrm{e}^{\mathrm{j} \omega_{o}}, \hat{\beta}_{N}\right)=C_{1}\left(\mathrm{e}^{\mathrm{j} \omega_{o}}\right)+C_{2}\left(\mathrm{e}^{\mathrm{j} \omega_{o}}\right)
$$

where

$$
\begin{aligned}
C_{1}\left(\mathrm{e}^{\mathrm{j} \omega_{o}}\right)= & \lambda_{o}\left|H\left(\mathrm{e}^{\mathrm{j} \omega_{o}}\right)\right|^{2} R_{z^{o}}^{-T}\left(\mathrm{e}^{\mathrm{j} \omega_{o}}\right) \\
& \times \sum_{k=1}^{n_{\beta}}\left(\mathcal{B}_{k}^{\beta}\left(\mathrm{e}^{\mathrm{j} \omega_{o}}\right)\right)^{\mathrm{T}} \overline{\mathcal{B}_{k}^{\beta}\left(\mathrm{e}^{\mathrm{j} \omega_{o}}\right)} \overline{R_{z^{o}}^{-1}\left(\mathrm{e}^{\mathrm{j} \omega_{o}}\right)}
\end{aligned}
$$

and

$$
C_{2}\left(\mathrm{e}^{\mathrm{j} \omega_{o}}\right)=L^{T}\left(\mathrm{e}^{\mathrm{j} \omega_{o}}\right) \sum_{k=1}^{n_{\alpha}}\left(\mathcal{B}_{k}^{\alpha}\left(\mathrm{e}^{\mathrm{j} \omega_{o}}\right)\right)^{\mathrm{T}} \overline{\mathcal{B}_{k}^{\alpha}\left(\mathrm{e}^{\mathrm{j} \omega_{o}}\right) L\left(\mathrm{e}^{\mathrm{j} \omega_{o}}\right)}
$$

where $L\left(\mathrm{e}^{\mathrm{j} \omega_{0}}\right)$ is defined in $(31)$.

Proof: The result follows directly from Lemma 2.2 and Theorem 3.1 .

The expression (32) can be made more explicit for specific model structures. In particular, for the standard polynomial model structures Box-Jenkins and output errors it is possible to provide explicit expressions for the basis functions in terms of the pole locations of the input spectrum, and system and noise dynamics. We refer to Ninness and Hjalmarsson (2004) for details.

While (32) provides an explicit finite model order expression for the asymptotic covariance matrix of the frequency function estimate $T\left(\mathrm{e}^{\mathrm{j} \omega_{o}}, \hat{\beta}_{N}\right)$, it is possible to reveal more of the structure by considering some special cases. In the following we will specialize to the case when $\left\{x_{t}\right\}$ is a separable process and when the input $\left\{u_{t}\right\}$ is uncorrelated with the noise $\left\{e_{t}\right\}$.

Theorem 4.3 Let $\Psi$ and $\Lambda$ be as in Theorem 4.2 and let the conditions (i) - (iv) of Theorem 3.4 be fulfilled.

Then $C_{2}\left(\mathrm{e}^{\mathrm{j} \omega_{o}}\right)$ (defined in Theorem 4.2) is given by

$$
C_{2}\left(\mathrm{e}^{\mathrm{j} \omega_{o}}\right)=\left[\begin{array}{rr}
\tilde{C}_{2}\left(\mathrm{e}^{\mathrm{j} \omega_{o}}\right) & 0 \\
0 & 0
\end{array}\right]
$$

where

$$
\begin{aligned}
\tilde{C}_{2}\left(\mathrm{e}^{\mathrm{j} \omega_{o}}\right)=\frac{1}{4}\left(\frac{\mathrm{d}}{\mathrm{d} \alpha}\right. & \left.\log \Phi_{x}\left(\mathrm{e}^{\mathrm{j} \omega_{o}}\right)\right)^{\mathrm{T}} \widetilde{P}_{\alpha} \\
& \times\left(\frac{\mathrm{d}}{\mathrm{d} \alpha} \log \Phi_{x}\left(\mathrm{e}^{\mathrm{j} \omega_{o}}\right)\right)\left|G_{o}\left(\mathrm{e}^{\mathrm{j} \omega_{o}}\right)\right|^{2}
\end{aligned}
$$

where

$$
\widetilde{P}_{\alpha}=\left\langle\frac{G_{o}}{\sqrt{\lambda_{o}} H_{o}} R_{\Delta}, \frac{G_{o}}{\sqrt{\lambda_{o}} H_{o}} R_{\Delta}\right\rangle^{-1}
$$

Proof: See Appendix C.

Remarks: When $n_{\beta} \rightarrow \infty$ it holds that $P_{\alpha} \rightarrow \widetilde{P}_{\alpha}$, cf. Result 4.1. We make no formal proof of this statement here. Also note that $C_{2}$ above does not depend on the number of parameters in the linear model, $n_{\beta}$.

Returning to the discussion after Result 4.1, Theorem 4.3 shows that the numerical observation in Ninness and Gibson (2002) is correct, i.e., that the second term of the right-hand side of (27) improves the accuracy of the variance expression. Indeed, for finite model orders, under the assumptions of Theorem 4.3, the expression gives the exact increase of the asymptotic variance as compared to not estimating the non-linear part. 


\section{Simulations}

We will now study a numerical simulation example of the identification of the model

$$
\begin{aligned}
y_{t}= & G\left(q, \beta_{1}\right) X\left(u_{t}, \alpha\right)+H\left(q, \beta_{2}\right) e_{t} \\
=\frac{b_{1} q^{-1}+b_{2} q^{-2}}{1+f_{1} q^{-1}+f_{2} q^{-2}}\left(u_{t}+\right. & \left.\alpha_{1} u_{t}^{2}+\alpha_{2} u_{t}^{3}\right) \\
& +\frac{1+c_{1} q^{-1}}{1+d_{1} q^{-1}} e_{t}
\end{aligned}
$$

where the data is generated according to

$$
\begin{array}{r}
y_{t}=\frac{0.1548 q^{-1}+0.0939 q^{-2}}{1-0.9744 q^{-1}+0.2231 q^{-2}}\left(u_{t}+0.5 u_{t}^{3}\right) \\
+\frac{1}{1+0.9 q^{-1}} e_{t}
\end{array}
$$

with $\left\{e_{t}\right\}$ being white noise with variance $\lambda_{e}=0.01$. The input $\left\{u_{t}\right\}$ is a Gaussian process with $r_{u}(\tau):=$ $E\left[u_{t} u_{t-\tau}\right]=\lambda_{u} a^{\tau}$, which means that the spectrum is $\Phi_{u}\left(\mathrm{e}^{\mathrm{j} \omega}\right)=\lambda_{u} \frac{1-a^{2}}{1-\left.a \mathrm{e}^{\mathrm{j} \omega}\right|^{2}}$, where $a=0.5$ and $\lambda_{u}=0.8$.

Let $r_{u}(\tau)=E\left[u_{t} u_{t-\tau}\right]$ denote the auto-correlation of a signal $u$ and let $r_{u v}(\tau)=E\left[u_{t} v_{t-\tau}\right]$ denote the crosscorrelation between $u$ and $v$. The spectrum $\Phi_{u}\left(\mathrm{e}^{\mathrm{j} \omega}\right)$ is then given by $\sum_{k=-\infty}^{\infty} r_{u}(k) \mathrm{e}^{-j \omega k}$ and the crossspectrum $\Phi_{u v}\left(\mathrm{e}^{\mathrm{j} \omega}\right)$ is given by $\sum_{k=-\infty}^{\infty} r_{u v}(k) \mathrm{e}^{-j \omega k}$.

Let $x_{t}:=u_{t}+\alpha_{o} u_{t}^{3}$, where $\alpha_{o}=0.5$ corresponds to the true input in (38). Then for the higher order moments we have

$$
\begin{aligned}
r_{u^{2}}(\tau) & =\lambda_{u}^{2}+2 r_{u}^{2}(\tau) \\
r_{u^{3}}(\tau) & =9 \lambda_{u}^{2} r_{u}(\tau)+6 r_{u}^{3}(\tau) \\
r_{u^{2} u}(\tau) & =0 \\
r_{u^{3} u}(\tau) & =3 \lambda_{u} r_{u}(\tau) \\
r_{u^{3} u^{2}}(\tau) & =0 \\
r_{x}(\tau) & =\left(1+3 \alpha_{o} \lambda\right)^{2} r_{u}(\tau)+6 \alpha_{o}^{2} r_{u}^{3}(\tau) \\
r_{u^{2} x}(\tau) & =0 \\
r_{u^{3} x}(\tau) & =3 \lambda_{u}\left(1+3 \alpha_{o} \lambda_{u}\right) r_{u}(\tau)+6 \alpha_{o} r_{u}^{3}(\tau)
\end{aligned}
$$

Define $\widetilde{\Phi}_{3}\left(\mathrm{e}^{\mathrm{j} \omega}\right):=\sum_{k=-\infty}^{\infty} 6 r_{u}^{3}(k) \mathrm{e}^{-j \omega k}=6 \lambda_{u}^{3} \frac{1-a^{6}}{\left|1-a^{3} \mathrm{e}^{\mathrm{j} \omega}\right|^{2}}$. In this case, $\hat{\beta}_{1}$ will be asymptotically independent of both $\hat{\beta}_{2}$ and $\hat{\alpha}_{2}$ and the variance of $G\left(\mathrm{e}^{\mathrm{j} \omega}, \hat{\beta}_{1}\right)$ can be expressed as

$$
\operatorname{As} \operatorname{Var} G\left(\mathrm{e}^{\mathrm{j} \omega}, \hat{\theta}\right)=\lambda_{u} \Lambda^{T}\langle\tilde{\Psi} \Phi, \tilde{\Psi}\rangle^{-1} \bar{\Lambda}
$$

where

$$
\begin{array}{r}
\Lambda=\left[\begin{array}{c}
G^{\prime} \\
0
\end{array}\right], \quad \tilde{\Psi}=\left[\begin{array}{cc}
H_{o}^{-1} G^{\prime} & 0 \\
0 & H_{o}^{-1} G_{o}
\end{array}\right] \\
\Phi=\left[\begin{array}{cc}
\Phi_{x} & \Phi_{x u^{3}} \\
\Phi_{u^{3} x} & \Phi_{u^{3}}
\end{array}\right]=x_{1} x_{1}^{T} \Phi_{u}+x_{2} x_{2}^{T} \widetilde{\Phi}_{3} \\
x_{1}=\left[\begin{array}{c}
1+3 \alpha_{o} \lambda_{u} \\
3 \lambda_{u}
\end{array}\right], \quad x_{2}=\left[\begin{array}{c}
\alpha_{o} \\
1
\end{array}\right]
\end{array}
$$

This will be compared to the case when the linear models $G$ and $H$ are estimated from $y_{t}$ and $x_{t}^{o}$, in which case the asymptotic variance of $G\left(\mathrm{e}^{\mathrm{j} \omega}, \hat{\theta}\right)$ again is given by $(39)$, but this time with $\Lambda=G^{\prime}, \tilde{\Psi}=H_{o}^{-1} G^{\prime}$ and $\Phi=\Phi_{x}$.

The system (38) is simulated in Matlab and $N=1000$ samples of $y_{t}, u_{t}$ and $x_{t}^{o}$ are used to identify the Hammerstein model and the linear model described above. The identification is made with the University of Newcastle System Identification Toolbox (UNIT) with the default settings of the routine est.m.

The experiment is repeated 1000 times with different realizations of the noise $e_{t}$ and the sample variance (normalized with $N$ ) is compared with the asymptotic variance (39). The results are shown in Figure 2. The first thing to notice is that the variance of the linear part of the model increases significantly when also the static non-linearity is estimated. The increase of the variance is given by the term $\tilde{C}_{2}$ in Theorem 4.3 . The second thing to notice is that the asymptotic expressions for the variance are very accurate (in this example), also for finite sample sizes.

\section{Conclusions}

In this paper we have studied the accuracy of the Hammerstein model (1) in terms of the asymptotic covariance of an arbitrary function $J\left(\hat{\theta}_{N}\right)$. The parameter $\theta$ consists of $\beta$, the parameters of the dynamic linear models, and $\alpha$, the parameters of the static nonlinearity. The asymptotic covariance can be decomposed in two terms

$$
\operatorname{AsCov} J\left(\hat{\theta}_{N}\right)=C_{1}+C_{2}
$$

The first term $C_{1}$ corresponds to the covariance one would obtain if the nonlinearity was known and only the linear part of the model was estimated. The second term $C_{2}$ represents the increase in covariance when also the static nonlinearity has to be estimated. Expressions for $C_{1}$ and $C_{2}$ are given in terms of orthogonal projections in Theorems 3.1 and 3.4. For the case when $J(\theta)=T\left(\mathrm{e}^{\mathrm{j} \omega_{o}}, \beta\right)$, i.e. the frequency response of the linear models, Theorem 4.2 gives $C_{1}$ and $C_{2}$ in terms of 


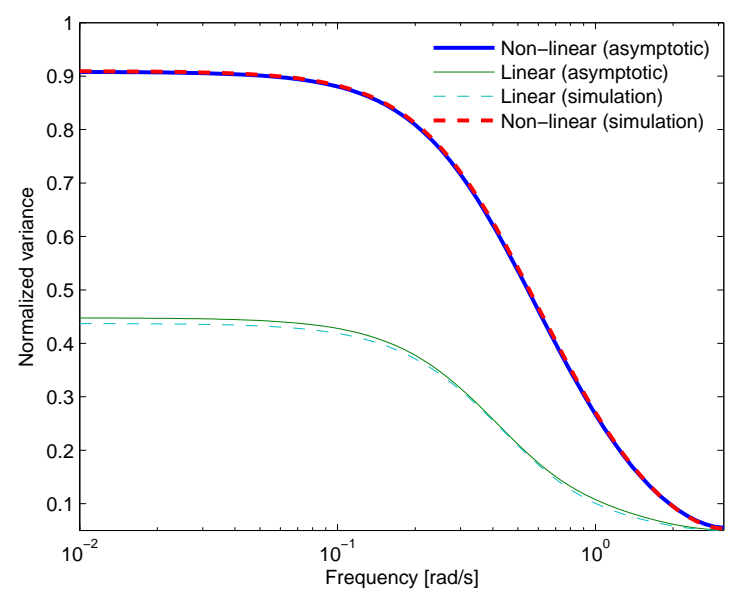

Fig. 2. Simulation results. The thick lines show the (normalized) variance of the linear part of the estimated Hammerstein model and the thin lines show the variance when the nonlinear part is known and only the linear part is estimated. The agreement with the asymptotic expressions (dashed lines) is very good.

orthonormal basis functions. Finally, under certain assumptions on the input and noise processes, $C_{2}$ is given a more explicit formulation in Theorem 4.3.

The expressions are valid for finite model orders and admit colored noise. In the general form there can be correlations between the input and the noise, but to derive most explicit expressions we must have them uncorrelated. A simulation study was used to evaluate the accuracy of the asymptotic expressions for finite sample sizes. The simulations show good agreement with the theoretic expressions.

Our results also explain the numerical observations in Ninness and Gibson (2002) that the second term of the right-hand side of (27) improves the accuracy of the variance expression when used for finite model orders, cf. the discussion after Theorem 4.3.

\section{Appendix}

\section{A An expression for $\Psi$}

The matrix $\Psi$ can be written as

$$
\Psi=\left[\begin{array}{cc}
\frac{1}{\sqrt{\lambda_{o}} H_{o}} T^{\prime} & 0 \\
0 & \frac{G_{o}}{\sqrt{\lambda_{o}} H_{o}} I
\end{array}\right]\left[\begin{array}{cc}
\Phi_{z^{o}} & \Phi_{z^{o} x^{\prime}} \\
\Phi_{x^{\prime} z^{o}} & \Phi_{x^{\prime}}
\end{array}\right]
$$

A spectral factorization is given by

$$
\left[\begin{array}{cc}
\Phi_{z^{o}} & \Phi_{z^{o} x^{\prime}} \\
\Phi_{x^{\prime} z^{o}} & \Phi_{x^{\prime}}
\end{array}\right]=R R^{*}, \quad R=\left[\begin{array}{cc}
R_{z} & 0 \\
\Phi_{x^{\prime} z^{o}} R_{z^{o}}^{-*} & R_{\Delta}
\end{array}\right]
$$

where $R_{\Delta}$ is a spectral factor of

$$
\Phi_{\Delta}:=\Phi_{x^{\prime}}-\Phi_{x^{\prime} z^{o}} \Phi_{z^{o}}^{-1} \Phi_{z^{o} x^{\prime}}
$$

This gives

$$
\Psi=\left[\begin{array}{cc}
\frac{1}{\sqrt{\lambda_{o}} H_{o}} T^{\prime} R_{z^{o}} & 0 \\
\frac{G_{o}}{\sqrt{\lambda_{o}} H_{o}} \Phi_{x^{\prime} z^{o}} R_{z^{o}}^{-*} & \frac{G_{o}}{\sqrt{\lambda_{o}} H_{o}} R_{\Delta}
\end{array}\right]
$$

\section{B Proof of Theorem 3.1}

Conditions (i) and (ii) implies, by Lemma 3.3, that

$$
\Phi_{x^{\prime} x^{o}}=M \Phi_{x^{o}}
$$

for some fix vector $M \in \mathbb{C}^{1 \times n_{\alpha}}$. Assumption (iii) gives that

$$
\Phi_{x^{\prime} z^{o}}=\left[\begin{array}{ll}
\Phi_{x^{\prime} x^{o}} & 0
\end{array}\right]=\left[\begin{array}{ll}
M \Phi_{x^{o}} & 0
\end{array}\right]
$$

and

$$
R_{z^{o}}=\left[\begin{array}{cc}
R_{x^{o}} & 0 \\
0 & \sqrt{\lambda_{o}}
\end{array}\right]
$$

Using these expressions in (16) gives

$$
\Psi=\frac{R_{x^{o}}}{\sqrt{\lambda_{o}} H_{o}}\left[\begin{array}{ccc}
G^{\prime} & \frac{\sqrt{\lambda_{o}}}{R_{x^{o}}} H^{\prime} & 0_{n_{\beta} \times n_{\alpha}} \\
M G_{o} & 0_{n_{\alpha} \times 1} & \frac{G_{o}}{R_{x^{o}}} R_{\Delta}
\end{array}\right]
$$

Finally, condition (iv) gives that the elements of the lower left $\left(n_{\alpha} \times 2\right)$-corner is spanned by the elements of the upper left $\left(n_{\beta} \times 2\right)$-corner, which means that the span of $\Psi$ is the same as the span of

$$
\frac{R_{x^{o}}}{\sqrt{\lambda_{o}} H_{o}}\left[\begin{array}{ccc}
G^{\prime} & \frac{\sqrt{\lambda_{o}}}{R_{x^{o}}} H^{\prime} & 0_{n_{\beta} \times n_{\alpha}} \\
0_{n_{\alpha} \times 1} & 0_{n_{\alpha} \times 1} & \frac{G_{o}}{R_{x^{o}}} R_{\Delta}
\end{array}\right]=\left[\begin{array}{l}
\Psi_{1} \\
\tilde{\Psi}_{2}
\end{array}\right]
$$

where the two block rows are orthogonal and the result of the theorem follows.

\section{Proof of Theorem 4.3}

We start with defining some block decompositions:

$$
\Psi=\left[\begin{array}{cc}
\Psi_{11} & 0 \\
\Psi_{21} & \Psi_{22}
\end{array}\right], \quad \gamma=\left[\begin{array}{ll}
\gamma_{1} & \gamma_{2}
\end{array}\right]
$$

Then it holds that

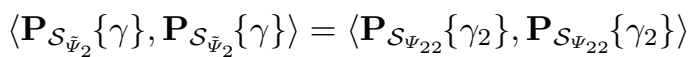


and furthermore, since $\langle\Psi, \gamma\rangle=\Lambda$,

$$
\begin{aligned}
\left\langle\Psi_{22}, \gamma_{2}\right\rangle & =-\left\langle\Psi_{21}, \gamma_{1}\right\rangle \\
\left\langle\Psi_{11}, \gamma_{1}\right\rangle & =T^{\prime}\left(\mathrm{e}^{\mathrm{j} \omega_{o}}\right) \\
& =\Psi_{11}\left(\mathrm{e}^{\mathrm{j} \omega_{o}}\right) R_{z^{o}}^{-1}\left(\mathrm{e}^{\mathrm{j} \omega_{o}}\right) H_{o}\left(\mathrm{e}^{\mathrm{j} \omega_{o}}\right) \sqrt{\lambda_{o}}
\end{aligned}
$$

One solution of $\gamma_{1}$ is

$$
\gamma_{1}=\sqrt{\lambda_{o} \overline{H_{o}\left(\mathrm{e}^{\mathrm{j} \omega_{o}}\right)}} R_{z^{\circ}}^{-*}\left(\mathrm{e}^{\mathrm{j} \omega_{o}}\right) \Psi_{11}^{*}\left(\mathrm{e}^{\mathrm{j} \omega_{o}}\right)\left\langle\Psi_{11}, \Psi_{11}\right\rangle^{-1} \Psi_{11}
$$

which gives

$$
\begin{aligned}
& \left\langle\Psi_{21}, \gamma_{1}\right\rangle \\
& =\left\langle\Psi_{21}, \Psi_{11}\right\rangle\left\langle\Psi_{11}, \Psi_{11}\right\rangle^{-1} \Psi_{11}\left(\mathrm{e}^{\mathrm{j} \omega_{o}}\right) R_{z^{o}}^{-1}\left(\mathrm{e}^{\mathrm{j} \omega_{o}}\right) H_{o}\left(\mathrm{e}^{\mathrm{j} \omega_{o}}\right) \sqrt{\lambda_{o}} \\
& =\mathbf{P}_{\Psi_{11}}\left\{\Psi_{21}\right\}\left(\mathrm{e}^{\mathrm{j} \omega_{o}}\right) R_{z^{o}}^{-1}\left(\mathrm{e}^{\mathrm{j} \omega_{o}}\right) H_{o}\left(\mathrm{e}^{\mathrm{j} \omega_{o}}\right) \sqrt{\lambda_{o}} \\
& =\Psi_{21}\left(\mathrm{e}^{\mathrm{j} \omega_{o}}\right) R_{z^{o}}^{-1}\left(\mathrm{e}^{\mathrm{j} \omega_{o}}\right) H_{o}\left(\mathrm{e}^{\mathrm{j} \omega_{o}}\right) \sqrt{\lambda_{o}} \\
& =G\left(\mathrm{e}^{\mathrm{j} \omega_{o}}\right)\left[\frac{\left.\Phi_{x^{\prime} x_{o}\left(\mathrm{e}^{\mathrm{j} \omega_{o}}\right)}^{\Phi_{x^{o} o}\left(\mathrm{e}^{\mathrm{j} \omega_{o}}\right)} 0\right]}{\text { C. }} 0\right]
\end{aligned}
$$

where the third equality follows from assumption (iv) and the last equality from assumption (iii).

Now

$$
\begin{aligned}
C_{2}\left(\mathrm{e}^{\mathrm{j} \omega_{o}}\right) & =\left\langle\mathbf{P}_{\mathcal{S}_{\Psi_{22}}}\left\{\gamma_{2}\right\}, \mathbf{P}_{\left.\mathcal{S}_{\Psi_{22}}\left\{\gamma_{2}\right\}\right\rangle}\right. \\
& =\left\langle\gamma_{2}, \Psi_{22}\right\rangle\left\langle\Psi_{22}, \Psi_{22}\right\rangle^{-1}\left\langle\Psi_{22}, \gamma_{2}\right\rangle \\
& =\left\langle\gamma_{1}, \Psi_{21}\right\rangle\left\langle\Psi_{22}, \Psi_{22}\right\rangle^{-1}\left\langle\Psi_{21}, \gamma_{1}\right\rangle \\
& =\left[\begin{array}{cr}
\tilde{C}_{2}\left(\mathrm{e}^{\mathrm{j} \omega_{o}}\right) & 0 \\
0 & 0
\end{array}\right]
\end{aligned}
$$

where

$\tilde{C}_{2}\left(\mathrm{e}^{\mathrm{j} \omega_{o}}\right)=\frac{\Phi_{x^{o} x^{\prime}}\left(\mathrm{e}^{\mathrm{j} \omega_{o}}\right)}{\Phi_{x^{o}}\left(\mathrm{e}^{\mathrm{j} \omega_{o}}\right)}\left\langle\Psi_{22}, \Psi_{22}\right\rangle^{-1} \frac{\Phi_{x^{\prime} x^{o}}\left(\mathrm{e}^{\mathrm{j} \omega_{o}}\right)}{\Phi_{x^{o}}\left(\mathrm{e}^{\mathrm{j} \omega_{o}}\right)}\left|G_{o}\left(\mathrm{e}^{\mathrm{j} \omega_{o}}\right)\right|^{2}$

Finally

$$
\frac{\Phi_{x^{\prime} x^{o}}\left(\mathrm{e}^{\mathrm{j} \omega_{o}}\right)}{\Phi_{x^{o}}\left(\mathrm{e}^{\mathrm{j} \omega_{o}}\right)}=\frac{1}{2} \frac{\mathrm{d}}{\mathrm{d} \alpha} \log \overline{\Phi_{x}\left(\mathrm{e}^{\mathrm{j} \omega_{o}}\right)}
$$

which concludes the proof.

\section{References}

Bai, E. W. (1998). An optimal two-stage identification algorithm for Hammerstein-Wiener nonlinear systems, Automatica 34(3): 333-338.

Bauer, D. and Ninness, B. (2002). Asymptotic properties of least-squares estimates of hammerstein-wiener models, International Journal of Control 75(1): 3451.
Cai, Z. and Bai, E.-W. (2011). Making parametric hammerstein system identification a linear problem, $A u-$ tomatica 47(8): $1806-1812$.

Crama, P. and Schoukens, J. (2001). Initial estimates of Wiener and Hammerstein systems using multisine excitation, IEEE Transactions on Instrumentation and Measurement 50(6): 1791-1795.

Ding, F. and Chen, T. (2005). Identification of hammerstein nonlinear armax systems, Automatica 41(9): 1479 - 1489.

Enqvist, M. (2005). Linear Models of Nonlinear Systems, $\mathrm{PhD}$ thesis, Linköping University.

Eskinat, E., Johnson, S. H. and Luyben, W. L. (2004). Use of Hammerstein models in identification of nonlinear systems, AIChE Journal 37(2): 255-268.

Goethals, I., Pelckmans, K., Suykens, J. A. K. and Moor, B. D. (2005). Identification of MIMO Hammerstein models using least squares support vector machines, Automatica 41(7): 1263-1272.

Greblicki, W. and Pawlak, M. (1986). Identification of discrete Hammerstein systems using kernel regression estimates, IEEE Transactions on Automatic Control 31(1): 74-77.

Hjalmarsson, H. and Mårtensson, J. (2007). A geometric approach to variance analysis in system identification: Theory and nonlinear systems, 46th IEEE Conference on Decision and Control, New Orleans, USA, pp. 5092-5097.

Hjalmarsson, H. and Mårtensson, J. (2011). A geometric approach to variance analysis in system identification, IEEE Transactions on Automatic Control 56(5): 983997.

Hjalmarsson, H., Mårtensson, J., Rojas, C. R. and Söderström, T. (2011). On the accuracy in errors-invariables identification compared to prediction-error identification, Automatica 47(12): 2704-2712.

Hunter, I. W. and Korenberg, M. J. (1986). The identification of nonlinear biological-systems - Wiener and Hammerstein cascade models, Biological Cybernetics 55(2-3): 135-144.

Lehmann, E. (1983). Theory of Point Estimation, John Wiley \& Sons, New York.

Liu, Y. and Bai, E.-W. (2007). Iterative identification of hammerstein systems, Automatica 43(2): 346 - 354 .

Ljung, L. and Caines, P. (1979). Asymptotic normality of prediction error estimators for approximative system models, Stochastics 3: 29-46.

Ninness, B. and Gibson, S. (2002). Quantifying the accuracy of Hammerstein model estimation, Automatica 38: 2037-2051.

Ninness, B. and Hjalmarsson, H. (2004). Variance error quantifications that are exact for finite model order, IEEE Transactions on Automatic Control 49(8): 1275-1291.

Ninness, B., Hjalmarsson, H. and Gustafsson, F. (1999). The fundamental role of general orthonormal bases in system identification, IEEE Transactions on Automatic Control 44(7): 1384-1406.

Nuttall, A. (1958). Theory and Application of the Sepa- 
rable Class of Random Processes, PhD thesis, MIT.

Vanbeylen, L., Pintelon, R. and Schoukens, J. (2008). Blind maximum likelihood identification of hammerstein systems, Automatica 44(12): 3139 - 3146 .

Wang, J., Zhang, Q. and Ljung, L. (2009). Revisiting hammerstein system identification through the twostage algorithm for bilinear parameter estimation, $\mathrm{Au}$ tomatica 45(11): 2627 - 2633.

Zhao, W.-X. (2010). Parametric identification of hammerstein systems with consistency results using stochastic inputs, IEEE Transactions on Automatic Control 55(2): 474-480.

Zhu, Y. (2000). Identification of Hammerstein models for control using ASYM, International Journal of Control 73(18): 1692-1702. 